\title{
Une plateforme pour l'enseignement des systèmes temps réel : le sas bancaire sécurisé
}

\author{
Eric Escande \\ Eric.Escande@grenoble-inp.fr \\ Grenoble INP - Grenoble Institute of Technology
}

\author{
Eric Zamaï \\ Eric.Zamai@grenoble-inp.fr \\ Grenoble INP - Grenoble Institute of Technology
}

RESUME : cet article présente un projet pédagogique centré autour d'une plateforme technologique. Cette dernière permet d'illustrer par la pratique les enseignements d'informatique industrielle et de méthodologie de conception des applications « temps réel » en deuxième année du cycle d'ingénieur de l'école pour l'énergie, l'eau et l'environnement (ENSE3 $\left.{ }^{1}\right)$. Le projet a pour objectif d'appliquer in fine le travail de spécification et de conception sur des systèmes informatiques modernes et diversifiés. Aussi, les thématiques traitées au sein de cet enseignement concernent :

- la méthode de spécification et de conception d'un système "temps réel" et

- les concepts fondamentaux de fonctionnement des systèmes industriels embarqués «temps réel » à base de processeurs (microcontrôleurs, processeurs de signaux ou processeurs généralistes), avec ou sans système d'exploitation multitâches.

Mots clés : dispositif pédagogique, applications temps réel, architecture multiprocesseurs, cycle en V, approche par projets, informatique industrielle, mécanismes d'interruption, outils industriels.

\section{Introduction}

De nos jours, de nombreux procédés industriels intègrent des systèmes à base de processeurs. Que ce soit dans les domaines des courants faibles (téléphonie mobile, gestion des réseaux de communication), des courants forts (contrôle commande de machines) ou encore dans les domaines de la production (automates programmables, superviseurs), ces systèmes se sont généralisés. Ils sont en outre disponibles à des coûts très variables.

Lors de la phase d'élaboration de l'application et afin de minimiser les temps de conception (par réduction des phases de tests des prototypes), les industriels utilisent de plus en plus des environnements de développement (ateliers de génie logiciel) qui manipulent des concepts « métiers » des ingénieurs [1] et qui ont pour objet de produire le code final de l'application, sa simulation, la documentation associée etc... comme c'est par exemple partiellement le cas dans l'aéronautique [2].

Pour enseigner aux étudiants l'ensemble de ces concepts, du fonctionnement de base d'un processeur avec son interfaçage au procédé physique (cours d'informatique industrielle) jusqu'à l'utilisation d'un outil informatique d'analyse et de programmation de haut niveau (cours de méthodologie de conception des systèmes temps réel), nous avons proposé une organisation pédagogique bâtie autour du cycle en $\mathrm{V}$ allant de la spécification du cahier des charges jusqu'à sa réalisation (partie programmation uniquement, donc hors partie mécanique et hors partie électronique) sur une maquette expérimentale. Cet enseignement proposé aux étudiants de deuxième année du cycle ingénieur s'appuie sur un ensemble d'outils industriels.

Partant d'un cahier des charges, les étudiants suivent la méthodologique proposée en faisant tout d'abord l'analyse de celui-ci, (outil Select Yourdon) puis en assure la spécification. Ils élaborent ensuite les programmes des systèmes temps réel, et les implantent enfin sur les cartes cibles, assurant par là la mise en œuvre technique du projet.
La partie réalisation s'appuie sur une maquette reproduisant le fonctionnement d'un sas bancaire sécurisé.

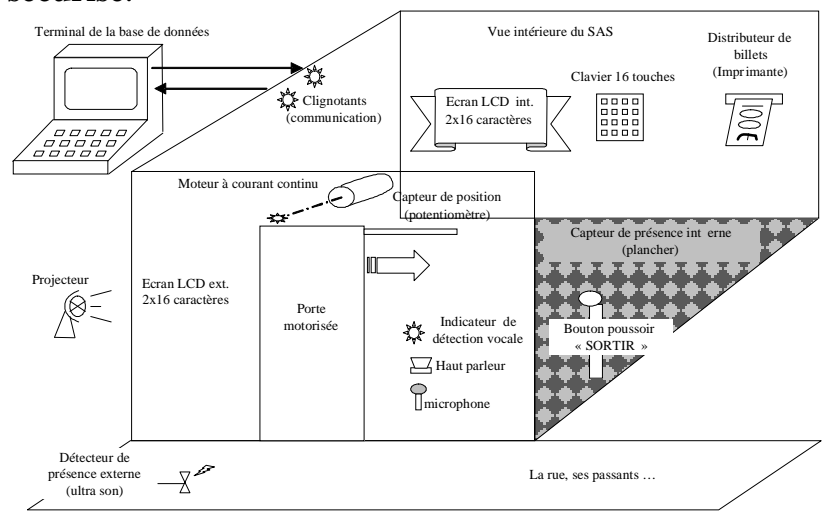

L'objet de cet article est de détailler plus particulièrement l'organisation des bureaux d'études attachés à ces enseignements.

L'architecture informatique de la maquette expérimentale mettant en œuvre le sas bancaire repose sur une structure à trois processeurs communicants (micro processeur, processeur de signal, micro contrôleur).

L'ensemble de ces trois systèmes informatiques "cibles" sont mis en œuvre à partir d'une salle équipée de postes de développement installés en réseau.

Voici la structure d'une partie des postes de la salle d'informatique industrielle :

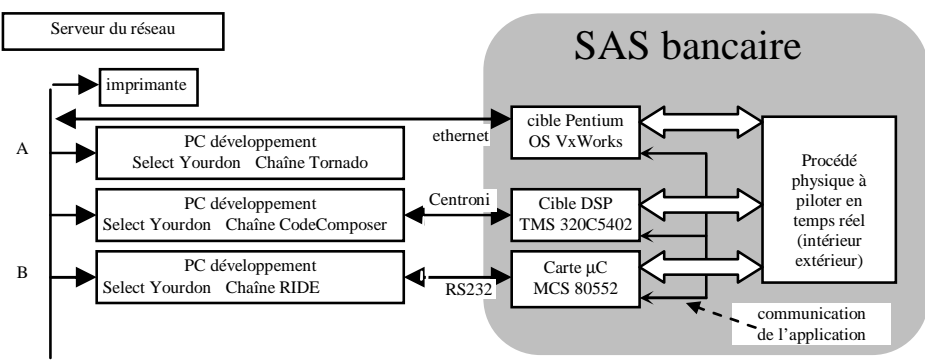

${ }^{1}$ http://ense3.grenoble-inp.fr/ 
Cet article se propose de présenter dans le détail les différents aspects de l'organisation pédagogique du projet. Ainsi, couverture thématique, approche par projets et thèmes, approfondissements à la carte ou encore adéquation à la problématique industrielle sont développés afin de montrer la pertinence pédagogique du projet proposé.

\section{Choix d'une organisation pédagogique}

Le volume horaire nécessaire pour traiter l'ensemble de ce projet pourrait-être très important compte tenu :

1- de la diversité des concepts liés au cours d'informatique industrielle (système avec ou sans OS multitâche, interruptions, interfaçage, etc.), mais également de la diversité des architectures matérielles industrielles mises en jeu, et

2- de notre volonté d'assurer le parcours complet du cycle en $\mathrm{V}$ [4] (de la spécification du cahier des charges à la mise au point de la solution sur la maquette finale).

Pour atteindre cet objectif pédagogique nous avons proposé de traiter cet enseignement selon l'organisation suivante :

- un cours de "méthodologie de conception des systèmes temps réel" 10h CM 4h TD

- un cours d' "informatique industrielle" $12 \mathrm{~h} \mathrm{CM}$ 4h TD

- un bureau d'études commun à ces deux matières d'un volume de $44 \mathrm{~h}$ : onze séances de $4 \mathrm{~h}$.

Le volume global (74h) est conséquent pour les 170 étudiants du deuxième cycle ingénieur (et pour l'institution) mais il est restreint compte tenu de l'ampleur du sujet. C'est pourquoi nous avons mis en place une organisation pédagogique spécifique, en particulier pour la mise en œuvre expérimentale du projet commun.

\subsection{Importante couverture thématique}

Nous venons de voir que dans ce projet d'enseignement, de nombreux thèmes pédagogiques sont à traiter : la méthodologie de spécification et de conception d'une application tempe réel (dont le cycle en V) et les éléments techniques de fonctionnement d'un système réellement embarqué (système d'exploitation, tâches différées, tâches immédiates, apprentissage du fonctionnement des systèmes à base de processeur : cycle machine, registres internes).

La diversité technique des phases du projet conduit à utiliser plusieurs outils industriels de conception : un outil d'analyse méthodologique de haut niveau (Select Yourdon ${ }^{2}$ ) et divers outils de programmation (chaînes de développement Tornado de Wind River Systems ${ }^{3}$, Code Composer Studio ${ }^{4}$ de Texas Instrument et RIDE de Raisonance ${ }^{5}$ ) nécessitant la compréhension de mécanismes techniques de bas niveau (assembleur - configuration des circuits). Ceci est mis en œuvre sur du matériel technique varié ( $\mathrm{PC}$, carte $\mu \mathrm{C}$, carte $\mathrm{DSP}$ ) que les étudiants ne connaissent pas nécessairement au début du cycle de formation.

\footnotetext{
${ }^{2} \mathrm{http}: / / \mathrm{www}$. selectbs.com/adt/

${ }^{3}$ http://www.windriver.com/

${ }^{4}$ http://focus.ti.com/

${ }^{5} \mathrm{http}: / /$ www.raisonance.com/
}

Compte tenu du nombre de séances fixé pour cet enseignement, les étudiants sont très sollicités. Sur une plate-forme d'une telle ampleur, si nous souhaitons que les étudiants traitent l'intégralité du projet nous risquons d'obtenir de leur part un simple survol des notions et non une réelle compréhension de leur fonctionnement.

Par ailleurs il nous a semblé que du point de vue des étudiants comme de l'équipe pédagogique, la focalisation du travail expérimental sur une technologie donnée limite le suivi de l'évolution technologique industrielle (dans sa diversité).

La solution retenue et mise en œuvre à l'ENSE3 pour résoudre le dilemme précédent, tout en exploitant les fondements de la méthodologie enseignée, consiste à ne faire réaliser à chaque étudiant qu'une partie de l'application répondant au cahier des charges : les manipulations ne se font que sur une partie réduite du matériel.

La maquette a été décomposée en trois thèmes, illustrant différentes solution technologiques :

- thème $\mathrm{A}$ : système avec système d'exploitation multi tâches sur processeur généraliste (pentium),

- thème $B$ : système sans système d'exploitation multi tâches (à base d'interruptions uniquement) sur microcontrôleur,

- thème $\mathrm{C}$ : système sans système d'exploitation multi tâches (à base d'interruptions uniquement) sur processeur de signal.

Chacun des thèmes est également décomposé à nouveau en plusieurs sous parties. Chaque étudiant ne traite au final qu'une seule sous-partie du projet. $\mathrm{La}$ nécessaire réalisation de l'ensemble du projet est assurée par la multiplicité des groupes d'étudiants au sein d'une série de 24 et par une coordination des divers travaux de ces groupes (2 à 3 étudiants par groupe). Cette organisation de l'enseignement par gestion du projet découpé en thèmes (et sous parties) permet de gérer les similitudes d'approche méthodologique et les disparités techniques de mise en œuvre.

Nous allons voir que cette solution résout parfaitement le problème initial et qu'elle comporte de nombreux avantages.

\subsection{Approche par projet : les trois thèmes décomposés}

Pour ce qui concerne les aspects méthodologiques, tous les étudiants suivent une formation expérimentale identique : la méthodologie de spécification et de conception d'un système temps réel vue en cours est détaillée dans ses différentes phases sur un cas concret: l'analyse des besoins (SADT[6] : le point de vue du client ; phase traitée lors des Travaux Dirigés), les spécifications de l'application à concevoir (SART[5] le point de vue du concepteur) et le passage à la mise en œuvre (phases traitées en Bureaux d'Etudes). La disparité issue du découpage en thèmes (et sous parties) réside simplement dans la focalisation sur telle ou telle partie du cahier des charges.

Pour ce qui concerne l'aspect « informatique industrielle », les points d'apprentissage expérimental obligatoires concernent le fonctionnement des systèmes à tâches immédiates (interruptions / priorités), le fonctionnement d'une unité centrale (registres internes, pile etc...), la compréhension du principe de fonctionnement d'une chaîne de développement d'une application destinée à être 
implantée sur un système embarqué, la compréhension des différents niveaux de langage $(\mathrm{C}$, assembleur et langage machine) et les problèmes d'interfaçage d'un processeur avec les matériels industriels (procédés numériques et analogiques).

Le découpage en thème n'introduit ici qu'une limitation de couverture sur le projet : sur chacune des zones, ou sur chacun des thèmes, la technologie du processeur diffère. Mais les diverses chaînes de développement intègrent des fonctionnalités similaires permettant une illustration diversifiée de concepts communs.

Cependant, comme nous l'avons évoqué, chaque groupe d'étudiant n'est amené à traiter qu'une partie du projet.

Ainsi, les notions techniques du fonctionnement des systèmes micro programmés sont traitées pour tous les étudiants et l'apprentissage expérimental des mêmes concepts est toujours illustré sur un processeur embarqué pilotant un procédé réel.

\subsection{La décomposition en divers thèmes : similitude et différences}

Il nous a fallu répartir les différents éléments du procédé " industriel " (le sas bancaire) sur les trois systèmes à base de processeurs (PC, DSP et microcontrôleur).

Cette répartition a été guidée par des considérations pédagogiques. Les divers capteurs et des actionneurs ont été répartis "équitablement" de telle sorte que tous les groupes puissent traiter des problèmes analogues.

Ce choix de la répartition du matériel fixe implicitement la répartition des diverses fonctionnalités finales (spécifiées au sein du cahier des charges) sur les trois systèmes à base de processeurs. Or cette répartition des activités est normalement issue de l'analyse méthodologique qui est menée par les étudiants. Nous procédons donc ici à la réalisation de la solution a priori de la génération de celleci. Cependant, le travail dans cet enseignement étant avant tout de nature programmation (de l'application temps réel), une grande latitude est possible. Par ailleurs, il n'y a pas de solutions "miracles" au problème posé par le cahier des charges et seul le matériel est bloquant... le procédé devant exister avant que les étudiants ne rentrent en salle ! Nous avons donc conçu les maquettes de telle sorte qu'elles intègrent déjà diverses solutions matérielles. Ceci permet une adaptation de l'application finale aux solutions proposées par les étudiants.

Dans cette décomposition, nous cherchons par ailleurs à répartir sur chacun des trois thèmes les différents concepts suivants :

A- la réactivité d'un équipement en temps réel vis-à-vis des événements matériels :

- traitement par interruptions matérielles,

- le choix des priorités entre les événements déclenchant les interruptions (plusieurs interruptions),

- le traitement d'un événement par scrutation (nécessité d'une interface en entrée 1 bit).

B- l'interfaçage d'un système numérique à un procédé physique :

- signaux analogiques,

- signaux numériques (TOR),

- interfaces en entrée et en sortie.
On retrouvera donc sur chacun des trois thèmes un ensemble "équilibré" de ces situations "temps réel" industrielles :

\begin{tabular}{|c|c|c|c|}
\hline & Thème A VxWorks & Thème $\mathrm{B} \mu \mathrm{C}$ & Thème C DSP \\
\hline Plusieurs Interruptions & $\begin{array}{l}\text { IT Clavier (scanf) } \\
\text { IT liaison série DSP } \\
\text { IT liaison série } \square \mathrm{C}\end{array}$ & $\begin{array}{l}\text { IT MLI } \\
\text { IT CAN } \\
\text { IT série } \\
\text { IT clavier }\end{array}$ & $\begin{array}{l}\text { IT CAN } \\
\text { IT détection présence } \\
\text { IT détection vocale } \\
\text { IT timer }\end{array}$ \\
\hline $\begin{array}{l}\text { scrutation évènement } \\
\text { Port en entrée } 1 \text { bit }\end{array}$ & & $\begin{array}{l}\text { BP ouverture porte } \\
\text { Présence interne }\end{array}$ & $\begin{array}{l}\text { Indicateur de détection } \\
\text { vocale } \\
\text { présence externe }\end{array}$ \\
\hline $\begin{array}{l}\text { Interface analogique } \\
\text { Ent }\end{array}$ & & asservissement porte $\square(\mathrm{t})$ & Enregistrement voix \\
\hline $\begin{array}{l}\text { Interface analogique } \\
\text { Sor }\end{array}$ & & & annonces vocales \\
\hline Interface TOR en sortie & "Imprimante" & $\begin{array}{l}2 \text { bits A et B pour hacheur } \\
4 \text { bits Clavier }\end{array}$ & $\begin{array}{l}\text { bit Projecteur } \\
\text { bit ind. détect voc } \\
\text { logicielle } \\
\text { bits Com liaison série } \\
\text { bit activ ULTRA SON } \\
\text { bit Rétro éclairage }\end{array}$ \\
\hline $\begin{array}{l}\text { interface TOR en } \\
\text { entrée }\end{array}$ & "Imprimante" & $\begin{array}{l}\text { Bouton Poussoir } \\
\text { ouverture porte } \\
\text { Présence interne } \\
\text { Clavier }\end{array}$ & $\begin{array}{l}\text { Indicateur de détection } \\
\text { vocale } \\
\text { présence externe }\end{array}$ \\
\hline
\end{tabular}

Les outils de développement répondent par ailleurs aux critères suivants :

- la carte dite " cible" est extérieure au poste de développement afin de favoriser la compréhension de l'existence d'un poste de développement et d'un poste cible,

- la maquette est équipée d'au moins une paroi transparente afin que les étudiants puissent visualiser le système temps réel (processeur, circuits d'interfaçage etc...),

- la carte externe dispose d'un mode de fonctionnement autonome (programme mis en UV EPROM ou Flash EPROM ou démarrage sur disque dur). Cela permet de faire fonctionner le procédé physique indépendamment des postes de développement,

- la chaîne de programmation intègre un compilateur $\mathbf{C}$,

- le débuggeur permet la mise au point des programmes directement sur la carte cible par le biais d'une communication à distance (RS232 ou parallèle ou réseau Ethernet),

- le débuggeur permet de faire la mise au point dans le langage source, ici le $\mathrm{C}$.

Le tableau ci-dessous résume cette recherche d'équilibre entre les trois thèmes :

\begin{tabular}{|c|c|c|c|}
\hline & Thème A VxWorks & Thème $B \mu C$ & Thème C DSP \\
\hline Chaîne & Tornado & Ride & Code Composer Studio \\
\hline Cible déportée & PC pentium & Carte Xéva MCS51 & DSK 5402 \\
\hline transfert de l'application & réseau ethernet & liaison série RS232 & câble parallèle \\
\hline $\begin{array}{l}\text { Visualisation du } \\
\text { processeur }\end{array}$ & $\begin{array}{l}\text { Carte mère de PC } \\
\text { connue }\end{array}$ & $\begin{array}{l}\text { OUI (vitre) } \\
\text { 80C552 }\end{array}$ & $\begin{array}{l}\text { OUI (vitre) } \\
\text { TMS320C5402 }\end{array}$ \\
\hline Programmation en $\mathrm{C}$ & OUI & OUI & OUI \\
\hline assembleur & $\begin{array}{l}\text { Visualisation mixte (C- } \\
\text { ASM) des fichiers } \\
\text { sources }\end{array}$ & $\begin{array}{l}\text { Visualisation mixte (C- } \\
\text { ASM) des fichiers } \\
\text { sources. } \\
\text { Programmation } \\
\text { assembleur } \\
\end{array}$ & $\begin{array}{l}\text { Visualisation mixte (C- } \\
\text { ASM) des fichiers } \\
\text { sources. } \\
\text { Programmation } \\
\text { assembleur }\end{array}$ \\
\hline $\begin{array}{l}\text { débuggeur source en C } \\
\text { évolué (Points d'arrêt...) }\end{array}$ & OUI & OUI & OUI \\
\hline $\begin{array}{l}\text { accès à l'architecture du } \\
\text { processeur (registres, } \\
\text { pile, Code, mémoire, } \\
\text { Ports...) }\end{array}$ & $\begin{array}{l}\text { Tache debug OUI } \\
\text { EIP, EAX EBX etc... }\end{array}$ & $\begin{array}{l}\text { OUI } \\
\text { PC, ACC, DPTR... } \\
\text { Disassembly Dump } \\
\text { Watch } \\
\end{array}$ & $\begin{array}{l}\text { OUI } \\
\text { IP A B T } \\
\text { Memory Stack Code ... }\end{array}$ \\
\hline Outil de simulation & OUI & OUI & \\
\hline
\end{tabular}

Cette structure de maquette permet aux différents groupes de travailler de façon "identique" sur des thématiques similaires mais en présence d'outils différents sur un procédé expérimental commun et quasi identique.

\subsection{L'approfondissement "à la carte"}

Une fois l'application complète développée et testée, les étudiants consacrent une séance de travail à l'approfondissement de notions techniques plus pointues qui sont elles, soit spécifiques à l'un des thèmes, soit plus à même d'être appréhendées sur l'un d'eux.

Pour des étudiants à bac+4, nous quittons ici un enseignement du type "sujet de TP rédigé" : les étudiants 
choisissent des sujets qui leur tiennent à cœur et qu'ils vont traiter comme ils l'entendent : ils réalisent les essais, tests et manipulations qu'ils jugent opportunes. Une liste de sujets et un canevas d'étude est proposé aux étudiants si besoin est.

C'est donc l'occasion de voir des spécificités des systèmes d'informatique industrielle : sur le thème $B(\mu C)$ : notions de démarrage d'un calculateur, vecteurs d'interruptions, interfaçage et configuration des circuits numériques avec le procédé industriel. Sur le thème C (DSP) notions de temps de calcul et utilisation des unités de traitement spécifiques. Sur le thème A (OS multi tâches) utilisation d'outil graphique (WindView) d'analyse du comportement du code en temps réel.

\subsection{Le planning des bureaux d'études}

Compte tenu de l'organisation souhaitée présentée précédemment, les onze séances de bureau d'études ont été organisées de la manière suivante :

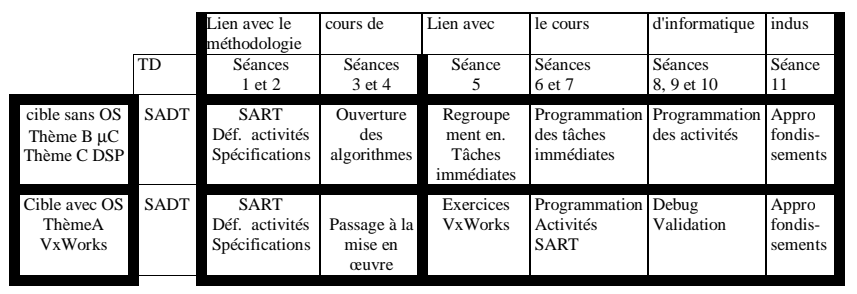

\subsection{L'organisation du travail par projet}

Nous proposons pour ces bureaux d'études, de responsabiliser les étudiants. Ceci se fait tout d'abord au travers du travail de type projet.

$\mathrm{Au}$ sein de ce projet par le découpage en thèmes chaque groupe prend sa part du tout pour laquelle il élabore sa solution. Chaque groupe mène cette partie sous sa responsabilité.

Chaque thème n'étant traité que par un seul groupe, les étudiants sont stimulés par les autres groupes pour l'aboutissement du projet final.

Ceci est un autre apport du découpage en thèmes.

La répartition des étudiants sur les divers thèmes est laissée à leur libre choix. Chaque étudiant peut orienter son travail en fonction de sa culture technique initiale : il complète judicieusement sa formation individuelle.

Notons que le choix du travail en divers groupes réunis au sein d'un projet commun favorise la compréhension de la phase d'intégration du cycle en $\mathrm{V}$.

Bien sûr, pour ne pas que l'ensemble du groupe soit bloqué par un binôme défaillant, cet enseignement prévoit un ensemble de filets et de garde-fous techniques.

\subsection{L'adéquation aux problèmes industriels}

Sur le " même " procédé pédagogique nous exposons des mises en œuvre destinées à des procédés industriels de différentes natures :

- procédés généralistes sans grosse puissance de calcul et peu onéreux. Réalisation de petites applications à base de microcontrôleurs.

- procédés orientés vers la production avec un fort interfaçage graphique, un fonctionnement en réseaux (superviseurs) sur des postes munis de systèmes d'exploitation multi tâches comme par exemple le pilotage d'axes de robots.

- ou des procédés nécessitant une très forte performance de calcul. Par exemple équipés de DSP.

Nous simulons sur une petite maquette des procédés industriels traditionnels :

- MLI hacheur (Pont en H) pour une partie de l'aspect énergétique.

- Asservissement numérique, capteurs TOR, supervision pour l'aspect automatique.

- Reconnaissance vocale pour l'aspect traitement du signal.

\subsection{La notion de temps réel}

Dans les systèmes industriels, les notions de temps réel ne sont pas toutes identiques. Nous avons souhaité traiter ces différences au sein de notre projet.

Nous disposons de cartes cibles avec ou sans système d'exploitation multi tâches, privilégiant ainsi une approche "bas niveau" avec gestion des interruptions, ou une approche plus fonctionnelle de la notion d'attente avec l'utilisation des tâches différées.

Les diverses cartes cibles ont des fréquences de fonctionnement variées (cycle à $1 \mathrm{~ms}$ pour le $\mathrm{mC}$, 10ns$100 \mathrm{MHz}$ pour le DSP ou carte mère du PC à $566 \mathrm{Mhz}$ pour le pentium) permettant ou non la mise en œuvre de certaines fonctionnalités (asservissement, traitement du son...)

Enfin au cœur de la cible munie du système d'exploitation multi tâches temps réel, l'influence du paramétrage de l'ordonnanceur des tâches est traité : concept de base de comptage du temps (TICKS) configurable au sein du noyau.

\section{La mise en œuvre technique}

La mise en œuvre technique de la plate-forme est organisée autour des systèmes informatiques suivants :

- le thème $B$ à base de microcontrôleur (chaîne RIDE) gère l'intérieur du sas bancaire. Il intègre des interfaces standards : écran clavier, Conversion analogique numérique et asservissement de la porte du sas.

- Le thème C à base de DSP (chaîne Code Composer Studio) gère l'extérieur du sas. Il intègre une partie traitement de la parole.

- Enfin, le thème A basé sur un PC standard (pentium chaîne Tornado VxWorks) centralise les communications et joue le rôle de banque. Il gère la base de données partagées en temps réel.

Voici l'un des cinq exemplaires disponible en salle de travail :

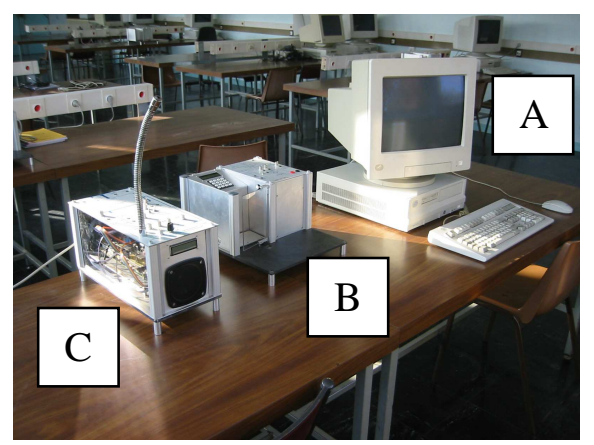




\section{Conclusion}

Enseigner les principes de base en informatique industrielle, convaincre de la nécessité d'une approche méthodologique pour le développement d'application temps réel complexes, initier les étudiants au travail en équipe, impliquer les étudiants dans leur propre processus d'apprentissage, tels étaient les grands défis à relever dans le cadre de ce projet. Sur un volume global de $74 \mathrm{~h}$ de formation ( $1 / 3$ théorique et $2 / 3$ pratique) centré sur une plateforme technologique réelle les étudiants appliquent l'ensemble des concepts théoriques enseignés tels qui le feraient dans le cadre industriels. De nombreux étudiants témoignent d'ailleurs de l'impact de cette formation dans leur recherche d'emploi et surtout de son réalisme vis-àvis des projets qu'ils mènent ensuite dans l'industrie.

Une telle formation par projet nécessite de la part de l'équipe pédagogique une maîtrise importante de toutes les phases du cycle en $\mathrm{V}$ et des outils de conception ainsi qu'une maîtrise des techniques dédiées à la programmation d'architectures à base de processeurs avec ou sans système d'exploitation multitâches temps réel.

Ceci occasionne une répartition des compétences au sein de l'équipe, une couverture distincte suivant les intervenants (thésards, permanents). Cela permet également dans le temps une évolution possible de cette couverture, au gré des souhaits de chacun.

Cette formation et la plateforme technologique développée à fait l'objet d'un transfert pédagogique dans le cadre des collaborations que nous avons avec le Vietnam, notamment au travers du Programme de Formation d'Ingénieur d'Excellence du Vietnam $\left(\right.$ PFIEV $\left.^{6}\right)$ au sein de l'Institut Polytechnique de Hanoi.

Dans un avenir proche fortement impacté par la réforme de notre offre de formation entrainant une perte du volume horaire attribué à cet enseignement, nous travaillons sur une réorganisation de la pédagogie tout en conservant le même thème d'étude du sas bancaire. Cette réorganisation vise en particulier, au travers d'une formation plus encadré, à faire traiter à chaque groupe d'étudiants des parties identiques, réparties sur l'ensemble des thèmes de la plateforme. Nous en aurons les premiers retours d'expérience prochainement.

\section{Remerciements}

Nous tenons ici à remercier l'équipe technique de l'école, et en particulier Mrs Didier et Thierry Blanc pour nous avoir suivi et accompagné dans le développement de la partie expérimentale très soignée de la plateforme.

\section{Bibliographie}

[1] "L'ingénierie dirigée par les modèles", Journée Neptune 2009 - Paris

[2] "Développement de logiciel temps-réel chez Dassault Aviation", Emmanuel Ledinot, Direction de la prospective$3^{\text {ième }}$ rencontres Ilia Tech - 21 juin 2000.

[3] «Principes des architectures de pilotage de procédés industriels », Coordination E.Zamaï, Techniques de l'Ingénieur, Traité "L'entreprise industrielle", AG3510, juillet 2007.

[4] «Génie logiciel, les méthodes », Patrick Jaulent, éd Armand Colin

[5] «Stratégies de spécifications des Systèmes temps réel : SART », D.J. Hatley et J.A. Pirbhai, éd. Masson

[6] «SADT, un langage pour communiquer», I.G.L. Technology, éd. Eyrolles

${ }^{6}$ http://www.pfiev.edu.vn/ 